\title{
Carotid Artery Prolapse and Myringocarotidopexy in Osteogenesis Imperfecta
}

\author{
Hassanin Abdulkarim 1*, Hassan Haidar1, Maryam Abdulraheem1, Ahmad Abualsoud1, \\ Ahmed Elsotouhy' ${ }^{2}$ A. Salam Alqahtani ${ }^{1}$ \\ ${ }^{1}$ Department of Otorhinolaryngology, Head \& Neck Surgery, Hamad Medical Corporation, Doha, Qatar \\ ${ }^{2}$ Department of Radiology, Hamad Medical Corporation, Doha, Qatar \\ Email: ${ }^{*}$ hassanainh2@hotmail.com
}

Received 15 June 2015; accepted 12 July 2015; published 15 July 2015

Copyright (C) 2015 by authors and Scientific Research Publishing Inc.

This work is licensed under the Creative Commons Attribution International License (CC BY).

http://creativecommons.org/licenses/by/4.0/

(c) (i) Open Access

\begin{abstract}
Osteogenesis Imperfecta is a rare genetic disorder of connective tissue that is caused by an error in collagen formation. The disease is characterized by abnormal bone fragility, osteopenia, blue discoloration of the sclerae and hearing loss. Chronic non-suppurative otitis media is frequent in Osteogenesis Imperfecta patients and usually attributed to Eustachian tube dysfunction due to cranial molding and deformities. In some cases of severe Osteogenesis Imperfecta, the fragile bone of the petrous carotid canal can be broken down by the pulsations of the carotid artery, this may result in prolapse of the carotid artery into the protympanum with resultant Eustachian tube obstruction and tympanic membrane retraction with adhesion to prolapsed carotid artery, a condition called myringocarotidopexy.
\end{abstract}

\section{Keywords}

Eustachian Tube, Carotid Artery, Osteogenesis Imperfecta, Chronic Otitis Media, Myringocarotidopexy, Hearing Loss

\section{Introduction}

Osteogenesis Imperfecta (OI) is a rare genetic disorder of connective tissue that is caused by an error in collagen formation. The disease is characterized by abnormal bone fragility, osteopenia, blue discoloration of the sclerae and hearing loss.

\footnotetext{
${ }^{*}$ Corresponding author.
}

How to cite this paper: Abdulkarim, H., Haidar, H., Abdulraheem, M., Abualsoud, A., Elsotouhy, A. and Alqahtani, A.S. (2015) Carotid Artery Prolapse and Myringocarotidopexy in Osteogenesis Imperfecta. International Journal of Otolaryngology and Head \& Neck Surgery, 4, 286-289. http://dx.doi.org/10.4236/ijohns.2015.44049 
Hearing loss is a common problem in patients with OI and it affects $30 \%-60 \%$ of patients [1]-[5]. The majority of OI patients usually develop a conductive hearing loss in the second to forth decade of life which gradually progress to mixed hearing loss [6]-[8].

Conductive hearing loss (CHL) in OI is often attributed to otosclerosis-like stapes fixation, ossicular discontinuity and chronic otitis media [2].

Chronic non-suppurative otitis media (COM) is frequent in young OI patients and usually attributed to eustachian tube (ET) dysfunction cranial molding and deformities [9].

In this report we present a 45-year-old lady with bilateral COM bilateral eustachian tube dysfunction caused by prolapsed Internal Carotid Arteries (ICA) with resultant adhesive otitis media and myringocarotidopexy.

\section{Case Report}

A 45-year-old Qatari female patient who is a known case of osteogenesis imperfecta, presented to the clinic complaining of reduced hearing in the right ear for long time, not associated with any other symptoms.

Examination of the ear revealed adhesive tympanic membrane with retrotympanic pinkish opacity in the right ear and retracted tympanic membrane in the left.

Pure tone audiogram showed bilateral moderate to severe mixed hearing loss. Impedance tympanometry showed bilateral flat (type B). Fiberoptic nasopharyngeal examination was done to rule out nasopharyngeal mass and was normal.

Temporal bone CT scan was ordered to discover the nature of the retrotympanic opacity. After that, CT angiogram was done and showed ET obstruction by a prolapsed ICA in the middle ear which confirmed our presumption of prolapsed ICA in the middle ear (Figure 1).

Patient was referred to the audiologist for hearing aid.

\section{Discussion}

Most of the ICA in the middle ear reported cases described as an aberrant ICA in the middle ear. Aberrant ICA is a variant of the ICA that passes through the middle ear. In this case we are presenting for the first time a bilateral prolapsed ICA in the middle ear. Presence of the carotid artery in the neck "CT angiogram" confirmed that the carotid artery in the middle ear is a prolapsed not aberrant carotid artery (Figure 2).

Patients with OI disease characterized by having brittle bones for that, the chance of getting spontaneous bone fractures is very high. Histopathologic studies of 8 temporal bones from 5 patients of OI, showed evidence of both deficient and abnormal ossification was found in the bony walls of middle ear and ossicles. Microfractures were found in the otic capsule and in the ossicles [9]. Depending on that, we assume that these microfractures can also happen in the ICA canal.

Computerized tomography (CT) of the temporal bone in an OI patient demonstrated otic capsule demineralization that appeared to progress as hearing diminished. Band-like areas of lucency were seen surrounding the cochlea, semicircular canals, the distal internal auditory canal, and oval window in an individual with hearing loss of mixed origin.
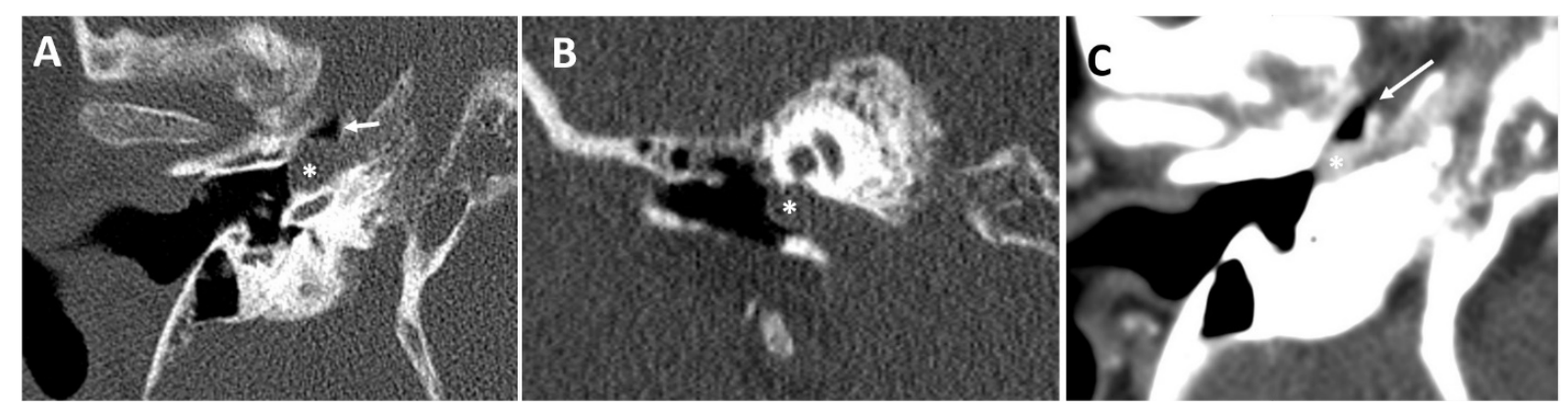

Figure 1. Right temporal bone Ct scan in axial (A), coronal (B), and after IV contrast in axial cut (C) showing dehiscence of the carotid canal and prolapse of the first genu of the petrous carotid artery $\left(^{*}\right)$ into the middle ear and encroaching upon the hypo-tympanic and protympanic spaces and occluding the bony part of Eustachian tube (arrow). IV contrast injection confirms the prolapse of the carotid into the Eustachian tube. Note the diffuse decrease in the bone density of the skull base bones in this patient with Osteogenesis Imperfecta. 


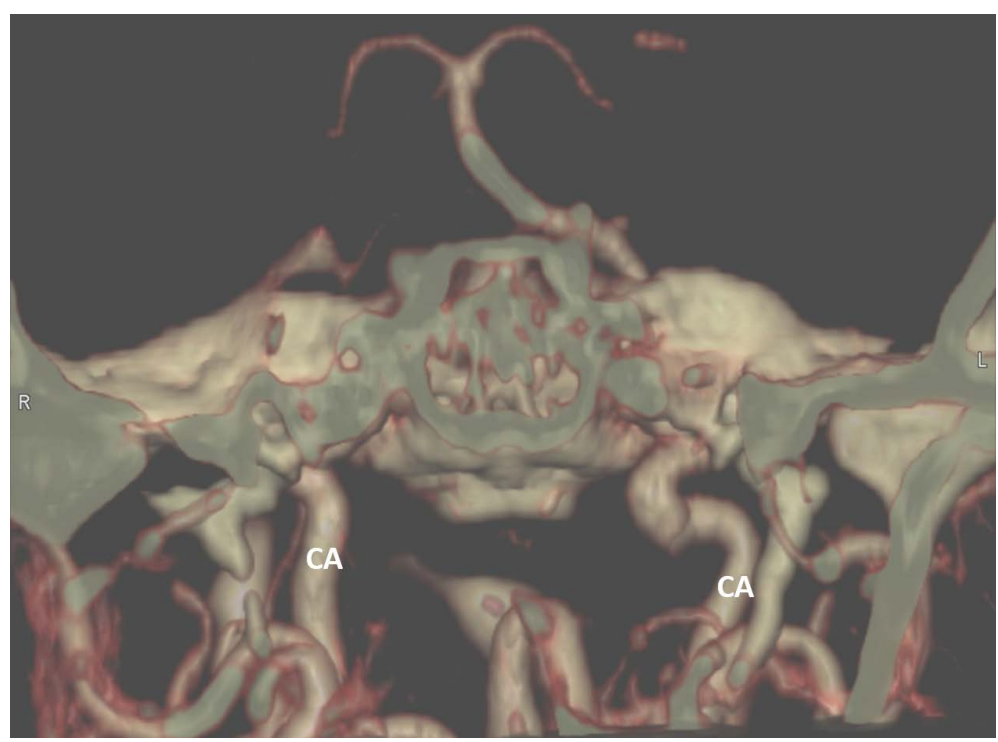

Figure 2. Colored 3D reconstruction of angio CT scan of skull base confirm the presence of both internal carotid arteries in the neck (CA).

Similarly, magnetic resonance imaging (MRI) examination of the otic capsule in type I OI demonstrated demineralized pericochlear lesions with soft tissue signal intensity and contrast enhancement

Postgadolinium T1-weighted MR imaging have shown symmetrical, band-like, homogeneously enhancing pericochlear areas, corresponding to the demineralization on CT, similar to retrofenestral or cochlear otosclerosis. High-resolution T2-weighted imaging may show irregularities in the outline of the labyrinthine and focal narrowing at the proximal basal turn of the cochlea, reflecting spongiotic foci.

The ICA wall itself is weak and thin due to collagen formation defect. Also the bony wall which separating the ICA canal and ET is naturally very thin. The thin wall canal, brittle bone and pulsation of the ICA all can contribute to the fracture of the canal and prolapse of the ICA.

This is the first report to show ICA prolapse as a cause of bilateral ET dysfunction, we suggest for patients with OI presented with AOM to do CT scan before myringotomy or ventilation tube insertion to avoid life threating/disastrous complications especially in those with craniofacial deformities or abnormalities.

The case at hand has already been approved by our institution's medical research and ethics committee at the research center with the reference number of (\#14332/14), the patient consent was taken for the publication of the case report and the figures and Authors and co-authors don't have any conflict of interest or financial interest to disclose.

\section{Disclosure}

The case at hand has already been approved by our institution's medical research and ethics committee at the research center with the reference number of (\#14332/14), the patient consent was taken for the publication of the case report and the figures and Authors and co-authors don't have any conflict of interest or financial interest to disclose. None of the authors was given an honorarium, grant or other form of payment to produce this paper. We declare no potential conflict of interests, real or perceived.

\section{Conclusion}

Computed tomography will guide us in identifying the cause of hearing loss in patients with osteogenesis imperfecta, also it plays a crucial role in avoiding a disastrous intervention like insertion of ventilation tube in such cases or craniofacial deformities. The role of temporal CT scan is major to rule out a nasopharyngeal mass in adult patients with a unilateral retracted tympanic membrane.

\section{References}

[1] Carey, M.C., Fitzgerald, O. and McKiernan, E. (1968) Osteogenesis Imperfecta in Twenty Three Members of a Kin- 
dred with Heritable Features Contributed by a Non-Specific Skeletal Disorder. Quarterly Journal of Medicine, 37, 437449.

[2] Stoller, F.M. (1962) The Ear in Osteogenesis Imperfecta. Laryngoscope, 72, 855-869. http://dx.doi.org/10.1288/00005537-196207000-00002

[3] Carruth, J.A., Lutman, M.E. and Stephens, S.D. (1978) An Audiological Investigation of Osteogenesis Imperfecta. The Journal of Laryngology \& Otology, 92, 853-860. http://dx.doi.org/10.1017/S0022215100086229

[4] Cox, J.R. and Simmons, C.L. (1982) Osteogenesis Imperfecta and Associated Hearing Loss in Five Kindreds. Southern Medical Journal, 75, 1222-1226. http://dx.doi.org/10.1097/00007611-198210000-00016

[5] Quisling, R.W., Moore, G.R., Jahrsdoerfer, R.A. and Cantrell, R.W. (1979) Osteogenesis Imperfecta: A Study of 160 Family Members. Archives of Otolaryngology, 105, 207-211. http://dx.doi.org/10.1001/archotol.1979.00790160041011

[6] Kuurila, K., Kaitila, I., Johansson, R. and Grenman, R. (2002) Hearing Loss in Finnish Adults with Osteogenesis Imperfecta: A Nationwide Survey. Annals of Otology, Rhinology \& Laryngology, 111, 939-946. http://dx.doi.org/10.1177/000348940211101014

[7] Pedersen, U. (1984) Hearing Loss in Patients with Osteogenesis Imperfecta. A Clinical and Audiological Study of 201 Patients. Scandinavian Audiology, 13, 67-74. http://dx.doi.org/10.3109/01050398409043042

[8] Stewart, E.J. and O’Reilly, B.F. (1989) A Clinical and Audiological Investigation of Osteogenesis Imperfecta. Clinical Otolaryngology \& Allied Sciences, 14, 509-514. http://dx.doi.org/10.1111/j.1365-2273.1989.tb00414.X

[9] Berger, G., Hawke, M., Johnson, A. and Proops, D. (1985) Histopathology of the Temporal Bone in Osteogenesis Imperfecta Congenita: A Report of 5 Cases. Laryngoscope, 95, 193-199. http://dx.doi.org/10.1288/00005537-198502000-00014 